\title{
BMJ Open Physicians' perceptions of followership in resuscitation in Japan and the USA: a qualitative study
}

\author{
Yoko Akamine (D) , ${ }^{1}$ Rintaro Imafuku, ${ }^{1,2}$ Takuya Saiki, ${ }^{1,2}$ Jannet Lee-Jayaram, ${ }^{3}$ \\ Benjamin W Berg, ${ }^{3}$ Yasuyuki Suzuki, ${ }^{1,2}$
}

To cite: Akamine $Y$, Imafuku R, Saiki T, et al. Physicians' perceptions of followership in resuscitation in Japan and the USA: a qualitative study. BMJ Open 2021;11:e047860. doi:10.1136/ bmjopen-2020-047860

- Prepublication history for this paper is available online. To view these files, please visit the journal online (http://dx.doi. org/10.1136/bmjopen-2020047860).

Received 10 December 2020 Accepted 22 July 2021

Check for updates

(c) Author(s) (or their employer(s)) 2021. Re-use permitted under CC BY-NC. No commercial re-use. See rights and permissions. Published by BMJ.

${ }^{1}$ Division of Medical Education Graduate School of Medicine, Gifu University, Gifu, Japan

${ }^{2}$ Medical Education Development Center, Gifu University, Gifu, Japan

${ }^{3}$ SimTiki Simulation Center, John A. Burns School of Medicine, University of Hawai'i at Manoa, Honolulu, Hawaii, USA

Correspondence to Dr Yoko Akamine; yokoakamine@gmail.com

\section{ABSTRACT}

Objectives While leadership is accepted as a crucial aspect of a successful resuscitation team, the role of followership has not been sufficiently explored. This study aims to explore physicians' perceptions of common favourable followership in resuscitation teams in two different countries.

Design A qualitative study with interviews and a reflexive thematic analysis.

Setting The authors individually interviewed critical care and emergency physicians whose clinical experience exceeded 6 years in Japan and the USA.

Participants A total of 18 physicians participated in a face-to-face, semistructured and in-depth interviews. Results Five themes and nine subthemes related to followership in resuscitation were identified. Under technical skills, two themes (being knowledgeable and skilled) and three subthemes (understanding guidelines/ algorithms, clinical course and being competent with procedural skills), were generated. Under non-technical skills, three themes (assuming roles, team communication and flattening hierarchy) and six subthemes (taking roles spontaneously, calm tone of voice, sharing information, closed-loop communication, respectful attitude and speaking up), were generated. Each generated theme involved commonly perceived favourable attributes of followership in resuscitation teams by experienced critical care and emergency physicians in both countries.

Conclusions This study clarified physicians' perception of common favourable followership attributes in resuscitation teams, both in Japan and in the USA. The results of this study shed light on followership-focused resuscitation education, where followership skills are generally underestimated.

\section{INTRODUCTION}

Resuscitation training is a standard element of healthcare providers' education globally. Previous research has identified nontechnical skills in dynamic healthcare teams as leadership, communication, task management and situational awareness. ${ }^{12}$ Of these, leadership is traditionally accentuated in resuscitation training. ${ }^{3} 4$ The 2015 American Heart Association guidelines state that implementing team and leadership training is reasonable as part of advanced life support

\section{Strengths and limitations of this study}

- This study is the first to explore physicians' perception of followership in resuscitation.

- We collected qualitative samples from experienced physicians who specialise in emergency medicine or critical care medicine in two different countries, Japan and the USA.

- Our reflexive approach to followership in resuscitation helped to enhance our analytical rigour.

- Our findings provide specific learning objectives for novices in resuscitation training.

- We only investigated physicians in this study, thus limiting the transferability of our findings to nurses and other healthcare professionals.

training. This intimates that in life support situations, a team leader with the ability to provide oversight of the team, provide guidance for specific tasks, and maintain a heightened level of situational awareness is needed. These guidelines, however, make no mention of followership. ${ }^{5}$ The 2015 European Resuscitation Council guidelines state that there is a need for not only leadership training, but also followership training in resuscitation education. However, the guidelines provide no further details regarding followership. ${ }^{6}$ Although a leader without followers would be nugatory to resuscitate a single patient, there has not been much focus on followership skills in this context. ${ }^{7}$

Followership in healthcare is being increasingly recognised to have a social influence on the leadership function within hospitals in general. ${ }^{7-9}$ Followership in a clinical setting is hard to define. ${ }^{10}$ The followership behaviour of team members is a complex element of teamwork that incorporates role, skills and social interactions that compliments, enhances and enables both team and leader functions. In a qualitative study interviewing medical trainees in the UK, positive followership attributes were shown to be achieving 
and embracing change, doing the job and working with others. ${ }^{11}$ Furthermore, the leader-follower dynamic is influenced by the hierarchy of healthcare situations. ${ }^{9} 11$ Multidisciplinary professionals who work within an implicitly strong hierarchy frequently experience conflicts. ${ }^{11-13}$ In such hierarchal organisations, proactive followership plays an important role in better team performance, job satisfaction and patient safety. ${ }^{10}$

Western countries have since started evaluating followership in the private business sector. Carsten identified effective followership qualities such as 'proactive attitude,' 'expressing opinions,' and 'being a team player'. ${ }^{14}$ Although this concept drew the attention of social psychologists, the word 'followership' was not seen in a favourable light. ${ }^{15}$ Kelley described the language of followership and its negative perception in English-speaking countries. ${ }^{16}$ This is in contrast with Asian countries, where the English word 'follower' did not originally exist, and paternalism and collectivism are lauded. ${ }^{17}$ Asian and Western countries have similarities and differences in both leadership and followership behaviours that are described in existing works. ${ }^{18-20}$ For example, a more direct leadership style and obedient followership are considered important in Japan because of the strongly paternalistic culture. In the USA, however, a less direct leadership style is neededespecially in highly professional jobs-because of strong individualism. $^{18}$

The followership-focused training model originated in the private business sector when Kelley published 'The Power of Followership' in 1992. ${ }^{21}$ Kelley explained that exemplary followers have high levels of both technical and non-technical skills. He asserted that the power of effective followership is underestimated and that it has a greater impact on team performance than leadership.

Exemplary followership in resuscitation, when time is limited, has not been described, and can be expected to differ from followership in private business and in nonemergency healthcare settings. From a global educational viewpoint, articulating common attributes of exemplary followership specific to a resuscitation team is imperative, because standardised resuscitation guidelines and training are already instituted worldwide. Moreover, resuscitation education is basically for novice trainees, therefore, emphasising leadership would not have an immediate impact on them because they would not yet be in a leadership role in real clinical resuscitation teams. Novice trainees would need followership training and its extensive feedback, which is more relevant to their practical work, and they can apply what they learnt immediately after training. We assumed that even in different countries or different local hospital cultures, there are common attributes for favourable followership in perceived successful resuscitation teams. We aimed to explore physicians' common perception of favourable followership in resuscitation teams because commonalities of followership between countries and local cultures are relevant to novice physician trainees' clinical practice and globalised resuscitation education, regardless of where they are trained.

\section{Research question}

This study aimed to explore experienced physicians' common perceptions of the attributes of favourable followership, specifically in resuscitation teams, in two different countries.

\section{METHODS \\ Design}

We followed the Standards for Reporting Qualitative Research recommendations. ${ }^{22}$ This study was based on the constructionist epistemological paradigm because the study aims to explore how physicians in two different countries perceive favourable followership in resuscitation. ${ }^{23}$ The qualitative data were collected in 18 face-to-face, semistructured, in-depth and open-ended interviews that were audiorecorded. All the data were inductively analysed. A reflexive thematic analysis was employed to elicit subjective meanings, which involved generative coding and theoretical interpretations by the research team. We chose Japan and the US based on the availability of the participants and the feasibility of the study because of the characteristics of our research team. In this study, we defined followership as abilities, skills, knowledge, action, attitude and behaviour of members of a team, except for the leader, based on Kelley's followership theory. ${ }^{21}$ Categorisation of technical and non-technical skills, which is common in describing teamwork skills ${ }^{24}{ }^{25}$ have been applied to our definition of followership. Therefore, we categorised abilities, skills and knowledge as technical skills, while action, attitude and behaviour, were categorised as non-technical skills.

\section{Participant selection}

Purposive sampling was directed to select physicians specialising in emergency medicine and critical care medicine-including adults, children, and newbornsand whose clinical experience exceeded 6 years. By 6 years, they would have completed their primary resident training period and would have been qualified as specialty physicians in emergency medicine and critical care medicine in both countries. Interviewees were selected based on their extensive clinical experience in resuscitation or in other emergency circumstances; these participants had experienced both followership roles and leadership roles during their careers. We intentionally selected some participants who had rich experience in both clinical and simulation-based resuscitation education. We recruited participants from our investigators' institute-related hospitals by asking for volunteers. Snowball sampling ${ }^{26}$ was employed to gather information and to access a specific group of people (mentioned earlier) working at university-based hospitals and tertiary-care children's hospitals in Japan and the USA. The gender 


\section{Box 1 Interview guide}

Preinterview questions: What is your gender? Age? Specialty?

1. Tell me about a time when you experienced the most successful crisis emergency team experience such as resuscitation. What kind of skill, behavior, or attitude did you see that was most successful?

- What happened? (series of events, behaviors, actions, etc)

- What was the patient outcome?

- What made him/her successful?

2. Tell me about a time when you experienced the worst (unsuccessful) crisis emergency team experience such as resuscitation? What kind of skill, behavior, or attitude did you see that was most unsuccessful or a failure?

- What happened? (series of events, behaviors, actions, etc)

- Is there any action or behavior that you think you or the person should have done at the time of the event?

- What was the patient outcome?

- What made him/her unsuccessful?

You can talk about yourself or someone you know. (Need specifics on the position of the person if they did not use themselves as an example.)

and age of the participants were balanced as much as possible in both countries.

\section{Patient and public involvement}

No patients were involved.

\section{Data collection}

Individual, face-to-face interviews were performed in a private room at the participants' hospitals or at the authors' institutes, with only the interviewer and interviewee present. The interviews followed a semistructured interview guide-with word modification - that was developed based on a previous study that explored followership (box 1). ${ }^{14}$ Their guide was designed to elicit participants' perspectives of followership and the attributes they believe make followers effective. In the current study, we intentionally used neutral terms, such as 'member' or 'teamwork' instead of 'follower' or 'followership' to minimise possible bias among interviewees towards those words. During the interviews, they were asked to reflect on their most and least successful experiences in real resuscitation or any other emergency teams.

There was no repeat interview. Each interview lasted approximately $40-50 \mathrm{~min}$. The first author (YA), who is Japanese and fluent in English, performed all interviews during the study period, January 2016-November 2017. Apart from three Japanese participants, the first author was unknown to the participants before undertaking this study. Further, the first author did not undertake any clinical or teaching activities locally alongside this research. All interviews were audiorecorded. Field notes were taken during the interviews to confirm the accuracy of the audio records immediately after each interview. None of the participants refused to answer the questions during the interview. Sample size and saturation were discussed in the analysis phase, regarding whether the data were rich enough to explore commonalities. Transcripts were not returned to participants for correction because the quality of the audio records was considered accurate enough in its details to be transcribed verbatim.

\section{Ethics}

Ethical concerns included maintaining the confidentiality of the sensitive information revealed in the interviews. All the participants were informed that the purpose of the study was to investigate exemplary followership attributes in the resuscitation team by asking clinicians' real experiences of teamwork in resuscitation or any other emergency situations; participants provided written consent in their own languages. They were informed that their data were confidential and that they could withdraw from the study at any time.

\section{Data analysis}

The digitally recorded audio data were transcribed verbatim in the original languages by professional technical personnel of commercial-based transcription services. Coding software (NVivo, V.12, QSR International, Massachusetts, USA) was used for organising the data. First, the transcripts were imported in NVivo in the original languages. Next, a reflexive thematic analysis that involves generative coding and theorisation, developed by Braun and Clarke,${ }^{2327}$ was applied. Although an inductive and latent approach was used to identify ideas of followership throughout the analysis, we incorporated sensitising concepts from existing followership theory by Kelley. ${ }^{21} \mathrm{~A}$ constructionist approach was applied because this study aimed to explore how physicians perceived successful and unsuccessful resuscitation experiences, and the authors' aim to identify common attributes for favourable followership in resuscitation in Japan and the US. We used six steps of thematic analysis. ${ }^{23}$ Japanese investigators (YA, TS, RI and YS) primarily initiated data analysis because they were academically proficient in both languages. Although this study was specifically on clinical resuscitation and emergency experiences, we assumed that TS, YS and RI could bring the perspectives of general healthcare providers and medical education researchers.

The transcribed data from both countries were analysed simultaneously. First, all the Japanese investigators read through the original verbatim transcribed data. Second, YA and YS individually performed selective coding in the original languages. Phrases or sentences that described followers' abilities, skills, knowledge, actions, attitude, behaviour, feelings and environment were labelled in the original language with a short description of the contents. Phrases or sentences that describe leaders' abilities, skills, knowledge, actions, attitude, behaviour, feelings and environment were not analysed, as leadership was not the main subject of this study. Third, all the Japanese investigators made a group agreement to combine codes to generate data-derived themes. At this stage, we generated all themes in the English language. After generating initial themes, YA and YS returned to the data and compared the themes to the data to ensure that the themes made sense, did not overlap, supported 


\begin{tabular}{lc}
$\begin{array}{l}\text { Table } 1 \text { Demographic and clinical characteristics of } \\
\text { participants }(\mathrm{N}=18)\end{array}$ & $\mathbf{N}$ \\
\hline Participant characteristics & \\
\hline Nationality & 9 \\
Japan & 9 \\
USA & \\
Gender & 10 \\
Men & 8 \\
Women & \\
Age & 5 \\
30 s & 10 \\
40 s & 3 \\
50 s & \\
Hospital characteristics & 8 \\
University-based hospital & 4 \\
Tertiary care general hospital & 6 \\
Tertiary care children's hospital & 3 \\
Specialty & 71 \\
Emergency medicine & 3 \\
Paediatric & \\
Critical care medicine & \\
Paediatric/neonatal & \\
\hline
\end{tabular}

the main theme, and examined whether there were other themes within the data. Next, all the Japanese investigators defined and named the generated themes and categorised them into technical and non-technical skills. In this phase, American investigators (JL-J and BWB) participated in the analysis. Code saturation ${ }^{28}$ was assessed by all investigators at this phase to determine whether 18 interviews were sufficient to generate high prevalence codes repeatedly and to capture themes alongside the aim of this study. As for the writing, the Standards for Reporting Qualitative Research recommendation were used for writing the report. ${ }^{22}$ After the themes were generated, we conducted a member check, a qualitative research technique in which a respondent's validation of the analysis confirms the authenticity of the interpretation. Participants confirmed that the generated themes were consistent with their narrative concepts.

\section{RESULTS}

Participants' demographics are shown in table 1.

From the thematic analysis of the interviews, five themes were generated as common favourable followership attributes in Japan and the USA. In technical skills, two themes and three subthemes were generated. In nontechnical skills, three themes and six subthemes were generated (box 2). After finishing all 18 interviews, we found that code saturation reached $80 \%$ within the first 14 interviews: 7 Japanese and 7 Americans. Another four

\section{Box 2 Generated themes}

\section{Technical skills \\ Being knowledgeable (11/18). \\ - Understanding guidelines/algorithms. \\ - Understanding clinical course of the patient. \\ Being skilled (10/18) \\ - Being competent with procedural skills. \\ Non-technical skills \\ Assuming roles (14/18) \\ - Taking roles spontaneously. \\ Team communication (17/18) \\ - Calm tone of voice. \\ - Sharing information. \\ - Closed-loop communication. \\ Flattening hierarchy $(13 / 18)$ \\ - Respectful attitude. \\ Speaking up.}

interviews added $20 \%$ to the codes, generating no new themes or subthemes.

\section{Technical skills}

\section{Being knowledgeable}

Most participants stated that having a basic knowledge of resuscitation, such as updated guidelines and algorithms, was a fundamental factor for favourable followers. Based on that knowledge, understanding the clinical course of the patient was considered favourable, meaning holding a big picture of the patient that enabled the team to identify what needed to be done and anticipate what would happen next in resuscitation.

\section{Understanding guidelines/algorithms}

Understanding guidelines and algorithms were considered an essential factor for the team to share the same purpose and to move smoothly without delay caused by too much conversation. Participants reported that cardiopulmonary resuscitation would be smoother when everyone knew what needed to happen during the first couple of minutes; this includes getting a backboard, performing chest compressions, and putting leads and monitors on without much conversation.

\section{Understanding the clinical course of the patient}

Understanding the patient's physiological clinical course, including treatments that had been provided and what would still be needed, was mentioned as favourable in both countries. Interviewees reported that understanding the clinical course provided members with a big picture of the patient and enabled them to anticipate what would happen next. Failing to anticipate further clinical events makes members ineffective, such as just doing unnecessary things, failing to prioritise actions or failing to find jobs that have not been completed.

\section{Being skilled}

Many participants described being skilled as being welltrained and experienced, and being able to correctly 
Table 2 Technical skills for followership in resuscitation themes, sub-themes, and quotes

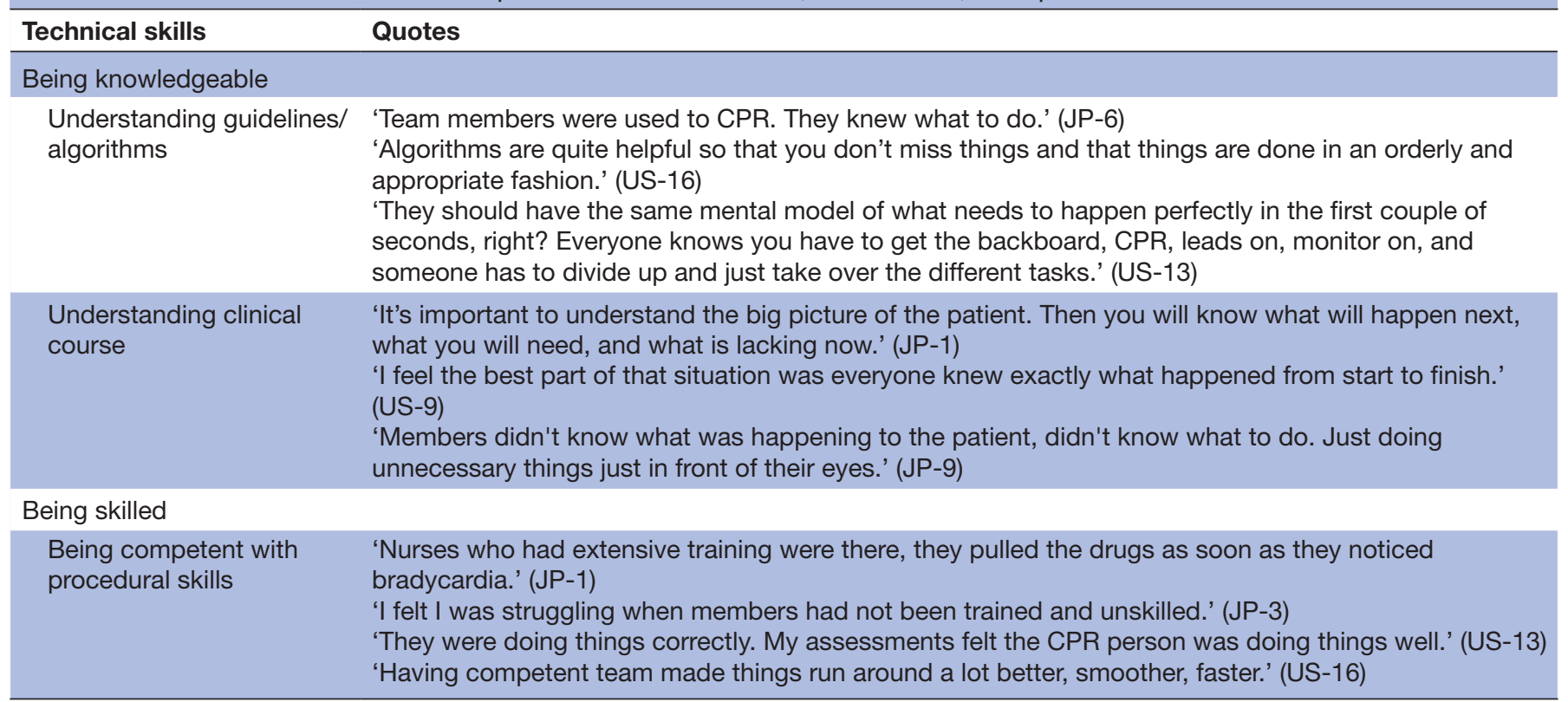

perform specific procedures required during resuscitation, which enabled the team to run smoothly and quickly. This was considered synonymous with being clinically independent concerning technical skills. The necessary skills differed according to the disciplines and included drawing up drugs, placing intravenous catheters, placing chest tubes or endotracheal intubation.

Quotes that support themes generated in technical skills are listed in table 2 .

\section{Non-technical skills}

\section{Assuming roles}

\section{Taking roles spontaneously}

Multiple roles exist in a resuscitation team. According to the participants, successful role dissemination within the team was crucial for successful resuscitation. Importantly, participants mentioned that exemplary followers took roles spontaneously without being told by the leader. Most interviewees considered that every member should think and act spontaneously, and not wait for the leader's directions to perform their roles according to their disciplines. In other words, accepting certain roles spontaneously and focusing on the completion of the responsibilities are important attributes for exemplary followers.

\section{Team communication}

Willingly communicating was mentioned as a desirable followership behaviour in resuscitation. The concept of communication referred to different behaviours and actions, including 'having a calm tone of voice', 'sharing information' and 'closed-loop communication'.

\section{Calm tone of voice}

Having a calm tone of voice, even when patients need emergency care and time is limited, was considered a highly positive attribute of followers. A chaotic tone of voice was specifically cited as a negative feature. Interviewees mentioned that the tone of voice tended to be louder because everyone tried to make their voice heard, resulting in a chaotic situation, which was perceived as a negative experience.

\section{Sharing information}

Participants discussed that it was essential to be verbal and let all the other members know what was happening and how they interpreted the situation. Our interviewees explained that if the team failed to share information, there would be a disruption in the chain of communication and considerable delay in making important decisions.

\section{Closed-loop communication}

Interviewees mentioned that closed-loop communication is a fundamental factor for favourable followers, and it enabled all team members to understand how far the resuscitation was proceeding. They described closed-loop communication as checking back and forth about the specific activity being assigned and completed within the team.

\section{Flattening hierarchy}

Hierarchy-related issues were often mentioned in the interviews. Interviewees described that hierarchy-related issues in resuscitation teams occurred concerning team members' age, years of clinical experience or occupations in both countries. Older or more experienced members or physicians may be considered superior to other members. The oldest physician was not always the leader of the team; however, team members assumed that they had to obey the older or more experienced physician. 


\section{Respectful attitude}

A respectful attitude was considered crucial for successful resuscitation teams, according to participants. A respectful attitude towards other professionals' backgrounds and their experience was deemed as favourable followership behaviour.

\section{Speaking up}

Speaking up was described as asking questions and making suggestions or recommendations, especially when the team experienced uncertainty. They recognised that speaking up in resuscitation was important for patient safety, yet difficult, because of the hierarchy related to members' age, years of experience, and occupations. Interviewees mentioned that it was difficult to communicate different ideas during resuscitation if members felt they were not listened to. There were some preferable ways to raise the potential problems in the conversation. For example, one should make recommendations at least three times as your professional responsibility for the patient, and use constructive and positive ways to ask questions, such as, 'Are you sure that's what you want to do?' or 'Did I hear you right?'

Quotes that support themes in non-technical skills are listed in table 3 .

\section{DISCUSSION}

This is the first study to explore common favourable followership attributes specific to resuscitation teams by interviewing resuscitation professionals from two different countries: Japan and the USA. Our interviews and analysis generated five themes and nine subthemes in followership in resuscitation. Our findings not only reinforce the existing followership theory ${ }^{141621}$ but also elaborate on the characteristic attributes for exemplary followers in resuscitation teams. Based on these findings, we can make recommendations to refocus resuscitation education by articulating exemplary followership attributes specifically for resuscitation teams.

Table 3 Non-technical skills for followership in resuscitation themes, subthemes and quotes

\begin{tabular}{|c|c|}
\hline $\begin{array}{l}\text { Non-technical } \\
\text { skills }\end{array}$ & Quotes \\
\hline \multicolumn{2}{|l|}{ Assuming roles } \\
\hline $\begin{array}{l}\text { Taking roles } \\
\text { spontaneously }\end{array}$ & $\begin{array}{l}\text { 'I like people to move without the leader's direction. I think it's preferable that members know their natural roles } \\
\text { and do the job without waiting to be told.' (JP-2) } \\
\text { 'Simultaneously, everyone was just going into their roles. And the right people got to the right locations and } \\
\text { things were happening simultaneously in a contemporaneous manner.' (US-13) } \\
\text { 'Everyone started to do the same thing at the same time! Just like a kid's soccer game. There was no clear } \\
\text { sharing of roles.' (JP-5) }\end{array}$ \\
\hline
\end{tabular}

Team communication

Calm tone of 'When we had trauma patient, it was good because they were calm. There was a trauma team, no one was voice screaming. If someone heated up, then others calmed down the person.' (JP-4)

'I've seen people that are inappropriate, yelling at people, and things just don't happen as quickly, or just being very timid, unsure of themselves, and so then the rest of the team loses faith in them.' (US-16)

$\begin{array}{ll}\text { Sharing } & \text { 'I want my team members to be very verbal stating of what they are doing right now, and things has been done } \\ \text { information } & \text { or not done yet. For example, 'I am getting IV.' or 'I am ordering chest X-ray.', and so on.' (JP-2) } \\ & \text { 'I felt it was good to have three different teams working and communicating. We gave information as things were } \\ & \text { going right - for the part we were doing - I could see that all those parts were going well while each of the minor } \\ & \text { procedure was going on.' (US-13) }\end{array}$

Closed-loop 'For example, cross check what one is doing with each other, how far it is proceeding.' (JP-1)

communication 'There's always what we call closed link communications, if the leader said, 'Okay, I would like XYZ done.' And then, whoever it is will say, 'Okay, XYZ done.' So, having that back and forth feedback.' (US-15)

\section{Flattening hierarchy}

Respectful 'There was no one who was very dominant over anyone else. The ideas were very willingly shared among all the attitude provider regardless of their role. They were respectful. Like respect for their knowledge backgrounds, respect for their experience.' (US-10)

'I think it was problematic because there was not a good organization, but also kind of creating an environment where people are shut down.' (US-15)

Speaking up 'I guess the age or experience definitely has big influence on a team. If they really think about the patient, doctor's age does not matter. People should say what they needed to say even your age was younger than the leader. If the leader said no to your suggestion, but you had better idea, try the second time. If you tried three times and the leader still ignores, then it is a problem.' (JP-7)

'Then you have to let the leader know that because you don't want to do the wrong thing on a patient. If you think it is wrong, and you have to phrase that in such a way to say. 'Wait a second, are you sure that's what you want to do?' And l've seen that was done as well at a check back. That's really hard to say no to someone when you know it's potentially wrong, but it's your duty for the patient to say it's wrong.' (US-12) 
First, exemplary followers should have knowledge and job skills at a mastery level and perform their roles spontaneously, in keeping with Kelley's theory of followership. ${ }^{21}$ In a resuscitation team, this translates to members taking on specific roles that match their disciplines or capabilities, according to their training level. Brahmajee et al reported that effective resuscitation teams in topperforming hospitals defined clear roles and responsibilities of team members, before resuscitation or early during resuscitation. ${ }^{29}$ Many interviewees in our study mentioned that it was favourable for the leader not to assign every single role. Instead, they preferred that followers spontaneously took their natural roles and performed their tasks independently and correctly. Moreover, it is not desirable if multiple members to overlap in the same role at the same time. Participants indicated that exemplary followers did not have overlapping roles; instead, they would identify and take other roles that no one had been doing. There may be a gap between reality and traditional resuscitation education, which typically focuses on the leader's obligation to assign the roles. Previously reported assessment instruments have specific items for individual leadership and teamwork as a collective, but not for individual followers. ${ }^{24}{ }^{25}$ 30-32 Consequently, the leader receives intensive feedback for the assignment of roles; however, other learners do not receive individualised feedback for taking roles spontaneously. It is therefore clear that resuscitation training needs to include followership assessment measures to provide feedback to all learners. The development of a validated followership assessment instrument in resuscitation can be a further area of research.

Next, team communication was identified as a crucial skill. Team communication includes a variety of factors, such as what should be verbalised and how it should be said during a resuscitation. The most specific communication style for resuscitation teams was 'closed-loop communication'. This is a well-established way of communicating during resuscitation, in which there is a verbal response to every communication. In addition to closed-loop communication, which is the current best practice, ${ }^{33} 34$ there were two more sub-themes in team communication. What should be verbalised was related to the subtheme of 'sharing information'. Ideal followers should verbalise what activity they are performing to let other members know, as each individual is occupied by their tasks. At the same time, how the information should be communicated was another important subtheme: 'calm tone of voice'. The tone of voice is a key factor that reflects emotions in human communication. ${ }^{35}$ A preferable tone of voice for leaders has been reported as calm, clear and direct. ${ }^{36}$ Interviewees in this study preferred a followers' tone of voice to be calm and assertive, not chaotic. A calm tone of voice may influence team trust, which is essential for perceived successful resuscitation. Conversely, yelling might intimidate other members, resulting in a reluctance to take roles or make suggestions and unsuccessful resuscitation. Our findings indicate that controlling one's tone of voice seems to be a major issue and that this could be a vital area of training during resuscitation education. Voice training in resuscitation is a further area of research, which could be feasible by using audio or video, according to a previous study on communication training methods for healthcare professionals. ${ }^{37}$

Finally, the majority of interviewees from both countries experienced hierarchy-related issues. Hierarchyrelated issues have been persistently reported as a major experience in healthcare. ${ }^{38}$ Our findings identified hierarchical factors such as age, years of experience and being physicians, which all have a considerable impact on teamwork during resuscitations in both countries. Japanese interviewees described older and more experienced physicians as always giving orders instead of supporting the leading younger physicians. They further explained that this was due to the belief that the most senior physician should take control, regardless of their expertise. This is in keeping with Japanese culture, which has a higher power distance than the USA. ${ }^{40}$ Power distance is defined as the extent to which the less powerful members of organisations within a country expect and accept that power is distributed unequally. ${ }^{40}$ The US interviewees mentioned more positive experiences, indicating that team members had a more respectful attitude than Japanese interviewees. Regarding hierarchy-related issues, 'speaking up' was perceived as an important attribute of favourable followers by interviewees from both countries, which was similar to previously reported findings. ${ }^{41}$ Our study participants expressed that speaking up could contribute to patient safety. Speaking up is crucial to avoid potential medical errors, especially in complicated situations. ${ }^{42-44}$ However, speaking up behaviours have been reported to be psychologically uncomfortable for healthcare workers. ${ }^{10}{ }^{13}$ Japanese interviewees considered this behaviour as an important quality for exemplary followers, similar to US interviewees; however, they specified that it was difficult to do so in real life because of the relatively high power distance in Japanese culture. Acknowledging the impact of the cultural background on followership behaviours may help educators in their approach when teaching communication strategies during resuscitation training.

The findings in our study suggest that specific technical and non-technical skills are required for followers in resuscitation teams to function effectively. It is also noted that there may be an overlap between followership and leadership attributes, considering our results and those of previous a study. ${ }^{12}$ For example, an effective leader needs profound knowledge of resuscitation algorithms, explicit communication, hierarchy reducing attitude and asking questions that highlight perceived problems. As one interviewee described, learning how to follow is learning how to lead. This idea embodies the main concept behind followership: the 'forgotten key to leadership'. 


\section{Limitations}

There are limitations to this study. First, we had a small number of interviewees from two different countries. Additional themes could have been identified if we interviewed a larger participant group from culturally wider spheres. Second, we only interviewed physicians. Nurses and physicians may have different perspectives; therefore, if we had included different professionals in the resuscitation team, different themes might have emerged. Third, we did not explore whether exemplary followership attributes identified in this study correlated with the patient outcomes. Further study would be necessary to clarify these issues.

\section{CONCLUSION}

This study described physicians' perceptions of common favourable followership attributes in resuscitation teams both in Japan and the USA. The results of this study contribute to exemplary follower-centric skills and behaviours that should be focused on during resuscitation education internationally.

Acknowledgements We would like to thank Editage (www.editage.com) for the English language editing.

Contributors YA contributed to conceptualisation, methodology, resources, investigation, data curation, formal analysis, writing and visualisation of data. RI and TS contributed to methodology, validation, formal analysis and review and editing. J-LJ contributed to resources, formal analysis, review and editing. BWB contributed to resources, formal analysis, review and editing, supervision and project administration. YS contributed to methodology, validation, formal analysis, review and editing, supervision and project administration.

Funding The authors have not declared a specific grant for this research from any funding agency in the public, commercial or not-for-profit sectors.

Competing interests None declared.

Patient consent for publication Not required.

Ethics approval All the study participants provided written informed consent. This research was approved by Institutional Review Board at Gifu University Graduate School of Medicine on 9 July 2014. (\#26-99) and University of Hawaii Human Studies Programme on 3 August 2015. (\#23224).

Provenance and peer review Not commissioned; externally peer reviewed. Data availability statement № data are available.

Open access This is an open access article distributed in accordance with the Creative Commons Attribution Non Commercial (CC BY-NC 4.0) license, which permits others to distribute, remix, adapt, build upon this work non-commercially, and license their derivative works on different terms, provided the original work is properly cited, appropriate credit is given, any changes made indicated, and the use is non-commercial. See: http://creativecommons.org/licenses/by-nc/4.0/.

ORCID iD

Yoko Akamine http://orcid.org/0000-0003-3467-5789

\section{REFERENCES}

1 Flin R, Patey R, Glavin R, et al. Anaesthetists' non-technical skills. Br $J$ Anaesth 2010;105:38-44.

2 Andersen PO, Jensen MK, Lippert A, et al. Identifying non-technical skills and barriers for improvement of teamwork in cardiac arrest teams. Resuscitation 2010;81:695-702.

3 Hunziker S, Bühlmann C, Tschan F, et al. Brief leadership instructions improve cardiopulmonary resuscitation in a high-fidelity simulation: a randomized controlled trial. Crit Care Med 2010;38:1086-91.
4 Cooper S. Developing leaders for advanced life support: evaluation of a training programme. Resuscitation 2001;49:33-8.

5 Bhanji F, Finn JC, Lockey A, et al. Part 8: education, implementation, and teams: 2015 international consensus on cardiopulmonary resuscitation and emergency cardiovascular care science with treatment recommendations. Circulation 2015;132:S242-68.

6 Greif R, Lockey AS, Conaghan P, et al. European resuscitation Council guidelines for resuscitation 2015: section 10. education and implementation of resuscitation. Resuscitation 2015;95:288-301.

7 Mannion H, McKimm J, O'Sullivan H. Followership, clinical leadership and social identity. Br J Hosp Med 2015;76:270-4.

8 Baker SD, Mathis CJ, Stites-Doe S. An exploratory study investigating leader and follower characteristics at US healthcare organizations. J Manag Issues 2011:341-63.

9 Gordon LJ, Rees CE, Ker JS, et al. Leadership and followership in the healthcare workplace: exploring medical trainees' experiences through narrative inquiry. BMJ Open 2015;5:e008898.

10 Leung C, Lucas A, Brindley P, et al. Followership: a review of the literature in healthcare and beyond. J Crit Care 2018;46:99-104.

11 Barrow M, McKimm J, Gasquoine S. The policy and the practice: early-career doctors and nurses as leaders and followers in the delivery of health care. Adv Health Sci Educ Theory Pract 2011;16:17-29.

12 Hunziker S, Johansson AC, Tschan F, et al. Teamwork and leadership in cardiopulmonary resuscitation. J Am Coll Cardiol 2011;57:2381-8.

13 Sculli GL, Fore AM, Sine DM, et al. Effective followership: a standardized algorithm to resolve clinical conflicts and improve teamwork. J Healthc Risk Manag 2015;35:21-30.

14 Carsten MK, Uhl-Bien M, West BJ, et al. Exploring social constructions of followership: a qualitative study. Leadersh $Q$ 2010;21:543-62.

15 Freeman M. Dispelling the myths of Followership in nursing. Can J Nurs Res 2021:53:3-4.

16 Kelley RE. In praise of followers. Harvard Business Review Case Services, 1988: 142-8. http://www.kquattrin.com/uploads/2/5/8/7/ 25876455/kelley_1988.pdf

17 Aycan Z. Paternalism. In: Kim U, Yang KS, Hwang KK, eds. Indigenous and cultural psychology. International and cultural psychology. Boston: Springer, 2006: 445-66.

18 Dorfman PW, Howell JP, Hibino S, et al. Leadership in Western and Asian countries: Commonalities and differences in effective leadership processes across cultures. Leadersh Q 1997;8:233-74.

19 Dickson MW, Den Hartog DN, Mitchelson JK. Research on leadership in a cross-cultural context: making progress, and raising new questions. Leadersh Q 2003;14:729-68.

20 Ezziane Z, Maruthappu M, Gawn L, et al. Building effective clinical teams in healthcare. J Health Organ Manag 2012;26:428-36.

21 Kelley RE. The power of Followership. New York: Doubleday Business, 1992.

22 O'Brien BC, Harris IB, Beckman TJ, et al. Standards for reporting qualitative research: a synthesis of recommendations. Acad Med 2014:89:1245-51.

23 Braun V, Clarke V. Successful qualitative research: a practical guide for beginners. London: SAGE Publications Ltd, 2013.

24 Cooper S, Cant R, Porter J, et al. Rating medical emergency teamwork performance: development of the team emergency assessment measure (team). Resuscitation 2010;81:446-52.

25 Repo JP, Rosqvist E, Lauritsalo S, et al. Translatability and validation of non-technical skills scale for trauma (T-NOTECHS) for assessing simulated multi-professional trauma team resuscitations. BMC Med Educ 2019;19:40.

26 Naderifar M, Goli H, Ghaljaie F. Snowball sampling: a purposeful method of sampling in qualitative research. Strides Dev Med Educ 2017;14:e67670.

27 Braun V, Clarke V. Reflecting on reflexive thematic analysis. Qual Res Sport Exerc Health 2019;11:589-97.

28 Hennink MM, Kaiser BN, Marconi VC. Code saturation versus meaning saturation: how many interviews are enough? Qual Health Res 2017;27:591-608.

29 Brahmajee K, Guetterman TC, Harrod M. How do resuscitation teams at Top-Performing hospitals for in-hospital cardiac arrest succeed? A qualitative study. Circulation 2018;138:154-63.

30 Grant EC, Grant VJ, Bhanji F, et al. The development and assessment of an evaluation tool for pediatric resident competence in leading simulated pediatric resuscitations. Resuscitation 2012;83:887-93

31 Fletcher G, Flin R, McGeorge P, et al. Anaesthetists' non-technical skills (ants): evaluation of a behavioural marker system. $\mathrm{Br} J$ Anaesth 2003;90:580-8.

32 Herzberg S, Hansen M, Schoonover A, et al. Association between measured teamwork and medical errors: an observational study of prehospital care in the USA. BMJ Open 2019;9:e025314. 
33 El-Shafy IA, Delgado J, Akerman M, et al. Closed-loop communication improves task completion in pediatric trauma resuscitation. J Surg Educ 2018;75:58-64.

34 Salik I, Ashurst JV. Closed loop communication training in medical simulation. Treasure Island: StatPearls Publishing, 2020. https://www. ncbi.nlm.nih.gov/books/NBK549899/?report=reader\#!po=2.94118

35 Juslin PN, Laukka P. Impact of intended emotion intensity on cue utilization and decoding accuracy in vocal expression of emotion. Emotion 2001;1:381-412.

36 Kolehmainen C, Brennan M, Filut A, et al. Afraid of being "witchy with a ' $b$ '": a qualitative study of how gender influences residents' experiences leading cardiopulmonary resuscitation. Acad Med 2014;89:1276-81.

37 Gysels M, Richardson A, Higginson IJ. Communication training for health professionals who care for patients with cancer: a systematic review of training methods. Support Care Cancer 2005;13:356-66.

38 Janss R, Rispens S, Segers M, et al. What is happening under the surface? Power, conflict and the performance of medical teams. Med Educ 2012;46:838-49.
39 Weller J, Boyd M, Cumin D. Teams, tribes and patient safety: overcoming barriers to effective teamwork in healthcare. Postgrad Med J 2014;90:149-54.

40 Hofstede Insights. Country comparison. Available: https://www. hofstede-insights.com/country-comparison/japan,the-usa/ [Accessed 23 May 2020].

41 Kobayashi H, Pian-Smith M, Sato M, et al. A cross-cultural survey of residents' perceived barriers in questioning/challenging authority. Qual Saf Health Care 2006;15:277-83.

42 Schwappach DLB, Gehring K. Silence that can be dangerous: a vignette study to assess healthcare professionals' likelihood of speaking up about safety concerns. PLoS One 2014;9:e104720.

43 Morrow KJ, Gustavson AM, Jones J. Speaking up behaviours (safety voices) of healthcare workers: a metasynthesis of qualitative research studies. Int J Nurs Stud 2016;64:42-51.

44 Okuyama A, Wagner C, Bijnen B. Speaking up for patient safety by hospital-based health care professionals: a literature review. BMC Health Serv Res 2014;14:61. 